\title{
The Need of Wavelength Conversion in All-Optical Networks
}

\author{
Authors: Marco Listanti ${ }^{\circ}$, Massimo Berdusco ${ }^{\circ}$, Roberto Sabella* \\ •Università di Roma "La Sapienza", Dipartimento INFO-COM, \\ Rome, Italy \\ * Ericsson Telecomunicazioni, Research \& Development \\ Division, Rome, Italy \\ Reference e-mail: sabella@RD.tei.ericsson.se
}

\begin{abstract}
Different optical path accommodationn design algorithms are analysed and discussed. Approaches using a rearrangment of wavelengths in each link are also proposed. The results show a drastic reduction of wavelength converters in each node.
\end{abstract}

\section{I - INTRODUCTION}

An optical path is a semi-permanent connection between path end points utilising optical cross- connects (OXC). In the literature two different approaches supporting optical paths have been introduced: virtual wavelength path (VWP) and wavelength path (WP), depending on whether the signal is carried by the same wavelength during its travel throughout the network or is converted to another in midstream. There is a significant debate in progress about the need of translating the wavelength of a signal within a network.

Different papers [1-3] in the literature show that, in real network structures, the implementations with and without wavelength translations require almost the same number of wavelengths. On the other hand, it has been also demonstrated that WP scheme incurs larger optical cross-connect (OXC) system scale than VWP scheme $[4,5]$. 
Moreover, it has been shown that the difference between WP and VWP schemes increases as the number of wavelengths carried on each fiber increases. This means that, even if the overall number of wavelengths is almost the same for the two approaches, the technological limitations, expressed as a maximum number of wavelengths which can be carried on the same fibers, cause a meaningful difference between the VWP and WP schemes, in the number of ports of the OXC's.

The optical path accommodation algorithm is a crucial point for the construction of a cost effective network. In general, these algorithms are basically composed of two steps: i) searching of the path route; ii) assignment of the wavelengths. In the VWP only the first step is needed. In fact, wavelength assignment is a trivial issue since each network link is independent of each other. On the other hand, in the WP both the problems have to be solved simultaneously throughout the network.

If the number $M$ of wavelengths per fiber is limited, each link consists of multiple fibers, so a link is defined as a bundle of fibers between two adjacent

OXC's. Each OXC has two types of ports: one runs to adjacent OXC's in different nodes ("inter-office ports"), the other connects to the electrical crossconnects (DXC) in the same node and accommodates the adding/dropping paths terminated at the node ("intra-office ports"). It is desirable that optical path (OP) be set-up in the network so as to minimize the total number of ports required at each node, that is the wavelength utilisation efficiency in link must be maximised. Thus the objective function is to minimize the mean value of number of ports in the network: here the number of ports at each node is defined as the summation of the number of inter-office ports $\left(\mathrm{N}_{1}\right)$ and intraoffice ports $\left(\mathrm{N}_{2}\right)$ at the node:

$$
\mathbf{N}=\mathbf{N}_{1}+\mathbf{N}_{2}
$$

In this paper we report an analysis of the algorithms which allows the implementation of VWP and WP schemes, and compare their performances in terms of $\mathrm{N}, \mathrm{N}_{1}$, and $\mathrm{N}_{2}$. Then we propose a rearrangement algorithm which allows the performance of VWP scheme to be reached, with a strongly reduced total number of wavelength converters with respect to the one corresponding to VWP. This permit a significant reduction of the OXC costs. In section II and III the accommodation design algorithms corresponding to VWP and WP schemes, respectively, are briefly reported. In section IV we compare the performances of VWP and WP schemes, while in section V we report the novel rearrangement algorithm which we propose, together with some relevant results. Finally, in section VI some concluding considerations are reported.

\section{II - VWP ACCOMMODATION DESIGN ALGORITHM}

Let $A$ be the path request matrix, wherein the value of the entry $(i, j)$ indicates the number of paths connecting the node $i$ to the node $j(0 \leq i, j \leq N$, wherein $N$ 
is the number of network nodes). We suppose that $\mathrm{A}$ is symmetrical, i.e. if a path request exists from $i$ to $j$, there also exists a request from $j$ to $i$. The summation of all the elements of $\mathrm{A}$ is named Traffic Volume and corresponds to the total number of paths to be set up within the network. Finally, let $\mathrm{p}_{i}$ be the number of paths accommodated in the link $\mathrm{i}(0 \leq \mathrm{i} \leq \mathrm{NL}$, being NL the number of network links).

As above mentioned, in the VWP scheme, only the path routes have to be found. Once the routing has been established, the final number of paths per link $\mathrm{p}_{\mathrm{i}} \quad(0 \leq \mathrm{i} \leq \mathrm{NL})$ and the number of terminating paths per node $\mathrm{T}_{\mathrm{j}}(0 \leq \mathrm{j} \leq$ NL) have been also determined, so the inter-office port requirements for each link $\mathrm{N}_{1 \mathrm{i}}$ is directly determined by $\left\lceil\mathrm{p}_{\mathrm{i}} / \mathrm{M}\right\rceil$, whereas the number of intraoffice ports per node $\mathrm{N}_{2 \mathrm{j}}$ is determined by $\left\lceil\mathrm{T}_{\mathrm{j}} / \mathrm{M}\right\rceil$.

The algorithm, sketched in fig. 1, consists of the following steps.

STEP1 - The initial path routes are set-up path so as to minimize the summation of $\mathbf{p}_{i}$, for each path and the set $\{\mathrm{NL}\}$ of network links for which $\mathbf{p}_{i}=\max \left(\mathrm{p}_{\mathrm{i}}, 0 \leq \mathrm{i} \leq \mathrm{NL}\right)$ is determined. The links belonging to this set are called "maximum links", they correspond to those links which support the maximum number of paths.

STEP2 - We reroute the paths that contain the maximum number of maximum links. This rerouting is carried out so as to make lower the cardinality of the set $\{\mathrm{NL}\}$.

STEP3 - The most inefficient links are found; these links are those with the minimum value of $\mathbf{C}_{\boldsymbol{i}}=\mathbf{p}_{\boldsymbol{i}} \bmod \mathbf{M}$, i.e. those links characterised by the highest waste of frequencies.

STEP4 - The paths containing the maximum number of inefficient links are found: these are the candidates for the rerouting.

STEP5 - The paths determined in the previous step are rerouted by applying Dijkstra's algorithm, employing the weighting function $\mathbf{W}_{i}$ for the $\mathrm{i}$-th link $(0 \leq \mathrm{i}$ $\leq \mathrm{NL})$ :

$$
\mathbf{W}_{i}= \begin{cases}\mathrm{p}_{\mathrm{i}} & \left(\mathrm{C}_{\mathrm{i}}=0\right) \\ 1 / \mathrm{C}_{\mathrm{i}} & \left(\mathrm{C}_{\mathrm{i}} \neq 0\right)\end{cases}
$$

\section{III - WP ACCOMMODATIONN DESIGN ALgORITHM}

In the WP scheme, the wavelength assignment problem must be solved: first we optimise path routes, then we assign wavelengths separately. The algorithm, whose flow-chart is shown in fig. 2, involves the following steps.

STEP1 - Route each path using VWP accommodation design procedure.

STEP2 - Divide the obtained path routes into the minimum number of sets of routes (we call them "layers"), such that the links in each layer must be occupied by as many WP routes as possible and no WP routes in one layer share any link. Let $\mathrm{L}$ be the number of layers. 
STEP3 - Allot a layer number (1 L) randomly to each layer so that each number appears once.

STEP4 - Assign a wavelength ( $M$ is the number of wavelengths within a fiber) cyclically to each layer according to the layer number. Wavelengths are assigned as follows: $\lambda_{1}$ to the paths in layer \#1, $\lambda_{2}$ to the paths in layer \#2, $\lambda_{M}$ to layer \#M, $\lambda_{1}$ to layer $\# M+1, \lambda_{2}$ to layer $\# M+2, \ldots$ From this wavelength assignment, we determine the number of inter-office ports $\mathrm{N}_{1}$ and intra-office ports $\mathrm{N}_{2}$ for each node in the network.

STEP5 - Obtain the average number of total ports $\mathrm{N}$ in the networks, according to the wavelength assignment performed in STEP4.

STEP6 - Iterate STEP3 STEP5 a certain number of times, finding the optimal layer assignment that give the minimum average value of $\mathrm{N}$.

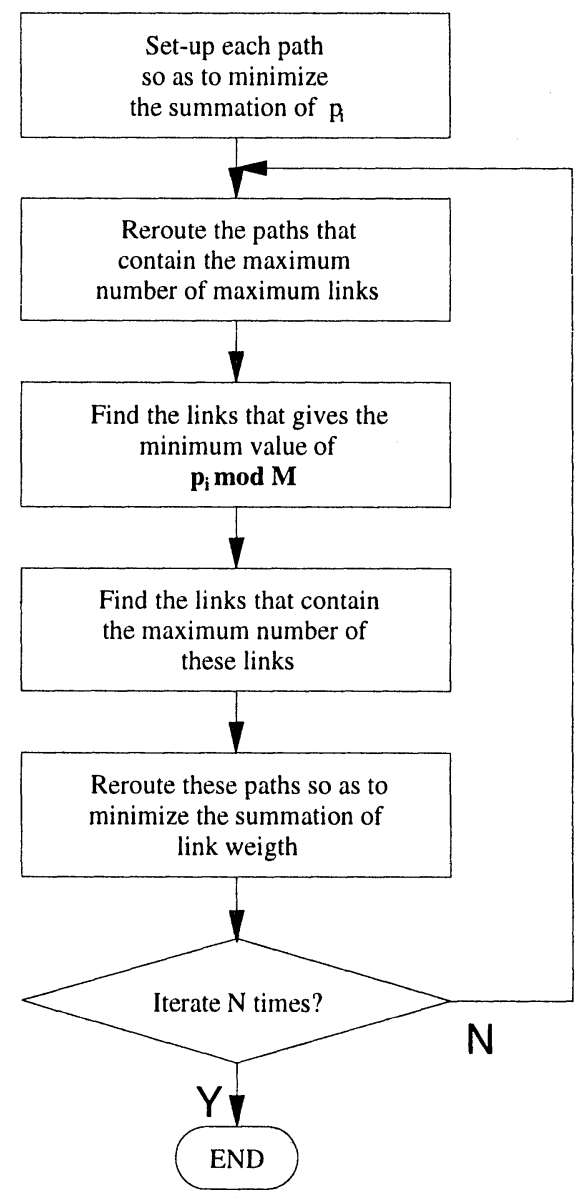

Fig. 1 - VWP accommodation design algorithm 


\section{IV - Performance Comparison Between the Different ACCOMmOdationn DESIgN Algorithms}

A relevant example of the performances of the two approaches reported so far, namely VWP and WP, are reported in figures $3 \sim 6$, considering a maximum number of channels per fiber $M$ equal to $4,8,16$ and 32 , respectively, in a $4 \times 6$ poligrid network.

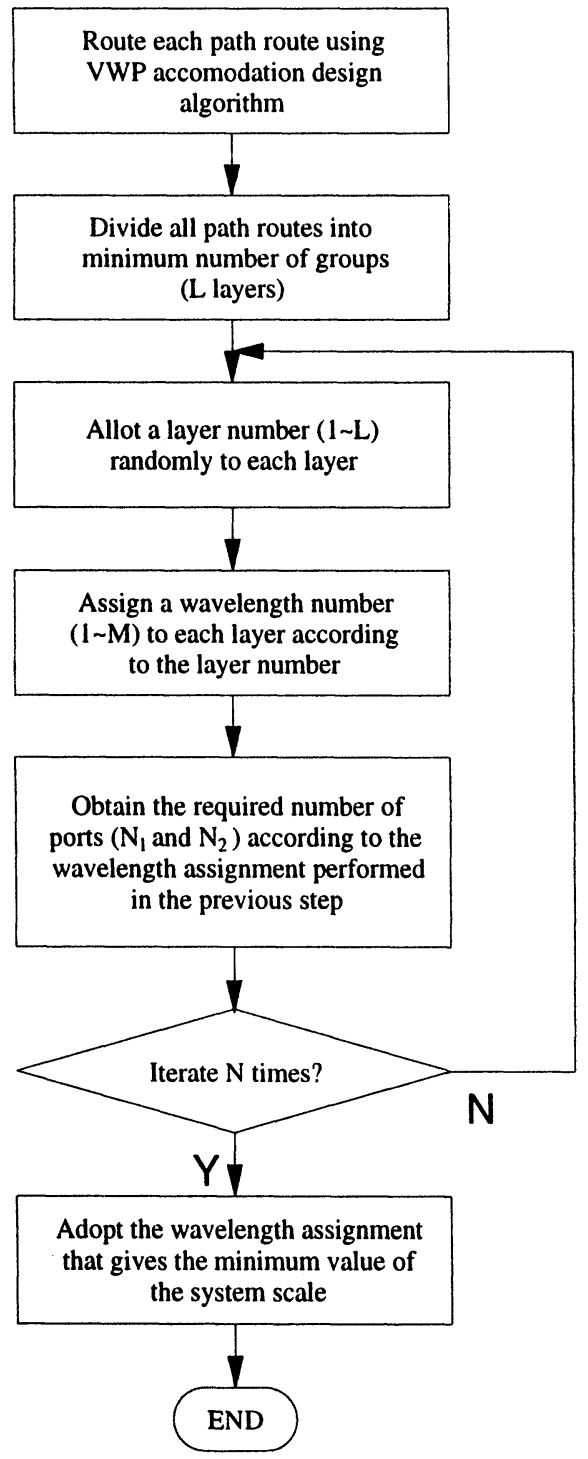

Fig. 2 - WP accommodationn design algorithm 
In particular the average number of ports per node is reported for different traffic volumes (total number of active paths). Each point is obtained averaging among many possible traffic patterns (obtained by randomly selecting terminating node pairs). It is worth observing that in any considered traffic volume, the average number of ports, in the case of VWP, is smaller than the corresponding one related to WP scheme. This is true either considering the total number of ports $\mathrm{N}$, or considering the inter-office ports $\mathrm{N}_{1}$ or the intraoffice ports $\mathrm{N}_{2}$.

In particular, comparing the different figures, it can be noticed that, when $\mathrm{M}$ becomes large, such a difference is much more evident.

The algorithms previously proposed show that the WP-based OXC requires a number of ports which is larger than that of VWP-based OXC, when the number of wavelengths multiplexed into a fiber is restricted. This means, altogether, that wavelength conversion allows the dimensions of the OXC to be reduced of a certain amount, which varies case by case.

However, we can imagine that a limited number of wavelength conversions, per each optical path, would allow the reduction of the OXC dimension towards the ones obtained through the VWP. In other words, it could be possible to obtain the same performance of the VWP scheme, in terms of medium number of ports per OXC, with less wavelength converters inside the nodes, with a consequent decreasing of the costs and complexity of the OXC themselves.

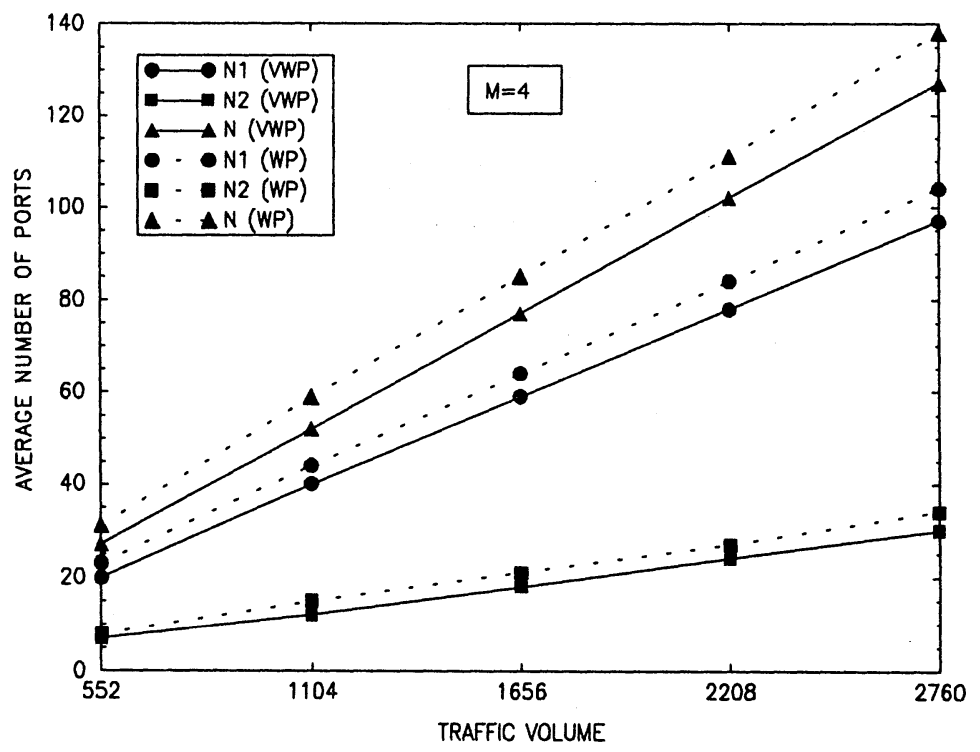

Fig. 3 - Average number of ports in the case of $M=4$ 
A first attempt, to investigate intermediate optical path schemes, was based on the following considerations. A long optical path, associated at a given wavelength, can be splitted in more than one sub-paths, each with a proper wavelength that can differ from each other. The length of each sub-path, expressed as the number of crossed OXC (hops), is limited to a given integer $b$. This means that the splitted paths are shorter than b hops. Each b hops a wavelength conversion occurs. Thus the path splitting generates some subpaths, to which wavelength assignment procedure is applied, as in WP algorithm. We can call such a scheme as b-WP, where b denotes the maximum sub-path length.

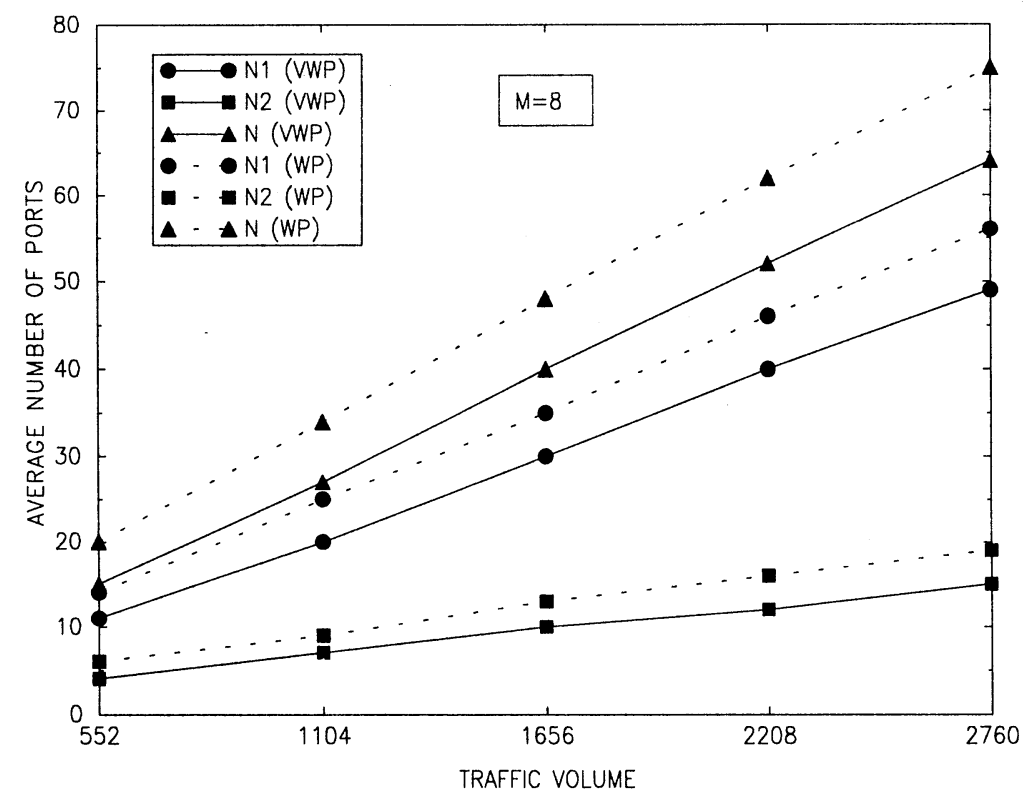

Fig. 4 - Average number of ports in the case of $M=8$

The results showed us that, as expected, the performance obtained through bWP schemes are somewhat intermediate between VWP and WP. However, such an approach is not effective for the following reason. The WP scheme causes that some fibers are scarcely utilised. This means that, in a general optical path, some links could be scarcely utilised. The b-WP approach does not consider when it is necessary to put wavelength conversion, but simply split in different sub-paths a main path. This is an uneffective way to proceed. A better approach is proposed in the following section. 


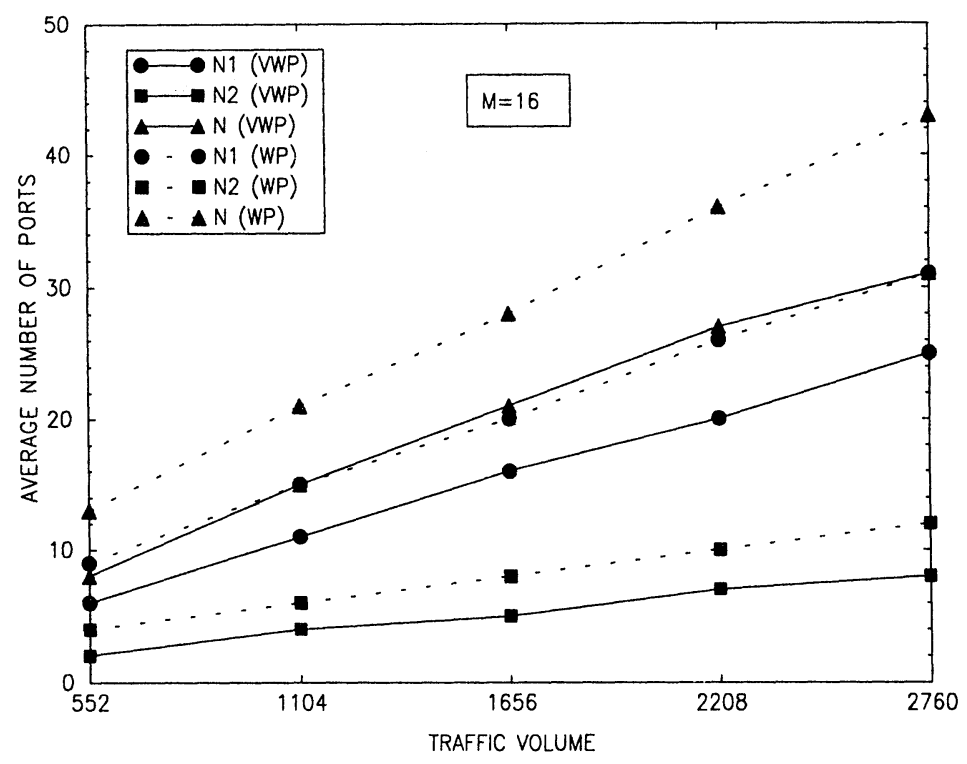

Fig. 5 - Average number of ports in the case of $M=16$

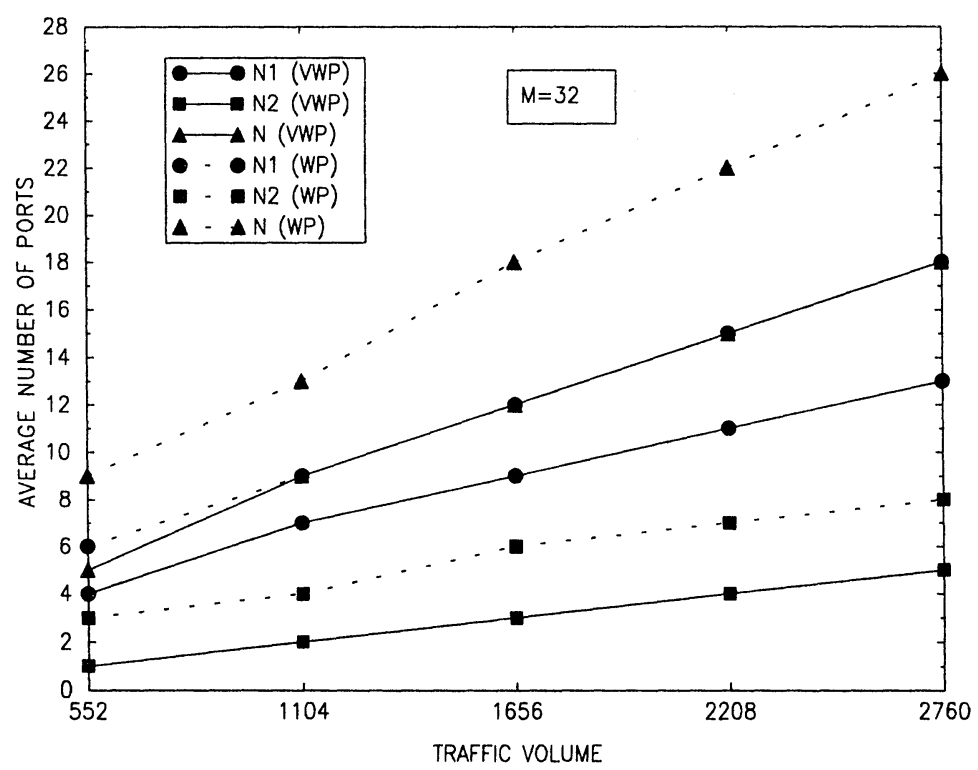

Fig. 6 - Average number of ports in the case of $M=32$ 


\section{V - REARRANGEMENT ALGORITHM}

We propose an algorithm that allows to rearrange the distribution of wavelengths in the fibers of each link, so as to eliminate in each link some fibers that are scarcely utilized: this procedure reduces the number of interoffice ports $\mathrm{N}_{1}$ in each node. Anyway a similar procedure can be applied to reduce the intra-office ports. The algorithm starts from wavelength assignment to optical paths in each layer (WP scheme): so it is possible to determine the mean value of $\mathrm{N}_{1}$ in the network. Moreover, we can observe that the wavelength assignment imposes, in each link, a number of fibers greater than the VWP scheme and induces a non efficient use of wavelengths in the fibers, since it's possible that a part of the wavelengths in each fiber have been assigned to some paths.

Let fibers of each link be numbered from 1 to $n$; let $M_{i}$ be the number of wavelengths that are used and $\mathbf{M}_{i}$ ' the number of non used wavelengths in the fiber $\# i\left(M_{i}+M_{i}^{\prime}=M\right.$, where $M$ is the number of wavelengths in each fiber). However if, in the fiber \#i of a certain link, the number of used wavelengths $\mathrm{M}_{i}$ is less than or equal to the number of non used wavelengths in previous fibers (i.e. $\mathrm{M}_{\mathrm{i}} \leq \sum_{\mathrm{j}=1}^{\mathrm{i}-1} \mathrm{M}_{\mathrm{i}}{ }^{\prime}$ ), it's possible to assign to each of $\mathrm{M}_{\mathrm{i}}$ paths of fiber \#i one of the non used wavelengths of other fibers: this can be realized by introducing , for each wavelength shifting, a converter in each node at the extremity of the link. If it's possible to shift the $M_{i}$ wavelengths, we can eliminate the fiber \# from the link. This reduction of the number of fibers in each link allows us to obtain in each node a value of $\mathrm{N}_{1}$ equal to VWP scheme, using a very small number of converters. So we can count the total number $\mathrm{K}$ of converters in the nodes of the network and determine the "Wavelength Conversion Percentage Amount" (WCPA), defined as:

\section{WCPA $=\mathrm{K} /\left(\mathrm{N}_{1} \mathrm{M}\right)$}

where $\mathrm{N}_{1}$ is the mean value of inter-office ports in WP scheme.

Such an algorithm allows the total number of wavelength converters to be drastically lowered. In fact, fig. 7 illustrates the WCPA versus the traffic volume, for different values of the number $M$ of wavelength carried by each fiber. Even in this case, each point of any curve is obtained by averaging the values obtained for many traffic patterns associated to a given traffic volume. 


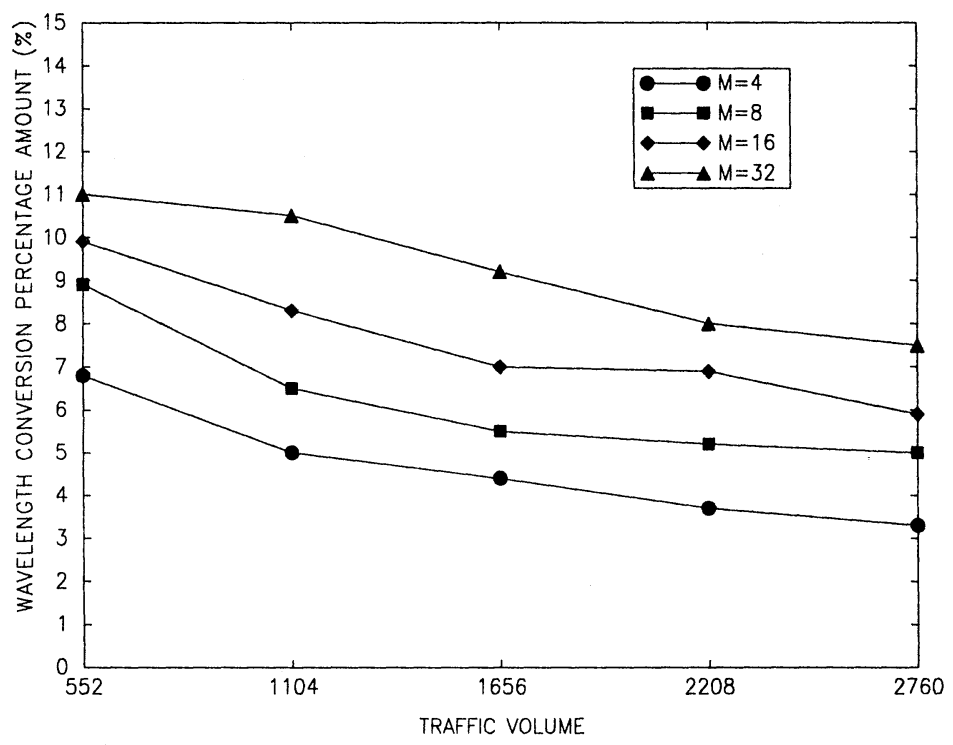

Fig. 7 - Wavelength Conversion Percentage Amount

The main result is that, adopting the considered approach, we can obtain the same performance of VWP scheme, using something like the $10 \%$ of the number of converters which are used with VWP scheme.

\section{VI - Conclusions ANd Perspectives}

Wavelength conversion allows the reduction of optical cross-connect system scale (number of ports). However, the employment of VWP scheme brings about a big number of wavelength converters inside the node, with a consequent increasing of complexity and costs. We have shown a possible algorithm that allows the number of converters to be drastically reduced, by locally solving the uneffective utilisation of the fiber links. Even this approach is not optimum and can be further improved by properly designing the network. This is the goal of further research. In any case the employment of a reduced number of converters demands for suitable OXC architecture, in which any number of converter can be used without altering the function of the node itself. 


\section{REFERENCES}

[1] R. Ramaswami, K.N. Sivarajan, "Routing and wavelength assignment in all-optical networks", IEEE/ACM Trans. on Networking, vol. 3, no. 5, pp.489-500, 1995.

[2] N. Nagatsu, et al., "Optical path accommodation designs applicable to large scale networks", IEICE Trans. Commun., Vol. E78-B, No.4, pp. 597-607, 1995.

[3] S. Baroni, P. Bayvel, "Analysis of restoration requirements in wavelengthrouted optical networks", Proc. NOC'96, pp. 56-63, 1996.

[4] N. Nagatsu, K. Sato, "Optical path accommodation design enabling crossconnect system scale evaluation", IEICE Trans. Commun.,Vol. E78-B, No. 9, pp. 1339-1343, 1995.

[5] N. Nagatsu, et al., "Optical path cross-connect system scale evaluation using path accommodation design for restricted wavelength multiplexing", IEEE J. Sel. Areas Commun., Vol. 14, No. 5, pp. 893-902, 1996. 